\title{
Laser capture microdissection: A tool for the molecular characterization of histologic subtypes of lung adenocarcinoma
}

\author{
VALENTINA DONATI ${ }^{1}$, CRISTIANA LUPI ${ }^{2}$, GRETA ALİ $^{2}$, VALENTINA CORSI $^{2}$, \\ ANDREA VITI $^{3}$, MARCO LUCCHI $^{3}$, ALFREDO MUSSI $^{3}$ and GABRIELLA FONTANINI ${ }^{2}$ \\ ${ }^{1}$ Section of Pathological Anatomy II, Azienda Ospedaliero-Universitaria Pisana, Pisa; \\ ${ }^{2}$ Section of Pathological Anatomy, Department of Surgery and ${ }^{3}$ Section of Thoracic Surgery, \\ Department of Cardio-Thoracic Surgery, University of Pisa, Pisa, Italy
}

Received May 5, 2009; Accepted June 25, 2009

DOI: 10.3892/ijmm_00000255

\begin{abstract}
The histologic heterogeneity of lung adenocarcinoma is well known. Many histologic subtypes have been described, and recently their prognostic and predictive value has emerged. Laser capture microdissection may aid in the isolation of cancer cells from distinct subtypes of lung adenocarcinoma, thus enabling the description of their specific molecular features. Characterization of epidermal growth factor receptor $(E G F R)$ mutations in histologic subtypes of lung adenocarcinoma has become an important issue. The purpose of this study was to analyze EGFR mutations in exons 18-21 in single histologic subtypes of lung adenocarcinoma after laser capture microdissection. A revision and reclassification of a series of 208 non-small cell lung cancers was conducted, and 62 adenocarcinomas with a total of 119 histologic component subtypes were identified. Laser capture microdissection of each subtype was performed. EGFR mutations in exons 18-21 were detected using polymerase chain reaction single-strand conformation polymorphism and direct DNA sequencing. EGFR mutations were detected only in 3 out of the 62 adenocarcinomas analyzed. Two adenocarcinomas harbored EGFR mutations in exon 19 (the E746T751 deletion VA insertion and the LREAT deletion) and one adenocarcinoma the EGFR exon 21 L858R missense point mutation. EGFR mutations were observed in all component subtypes. This suggests that, in a patient with lung adenocarcinoma, EGFR mutations are not associated with particular component histologic subtypes and probably occur at an early stage of tumorigenesis. Notably, 2 out of the 3 mutated adenocarcinomas had a bronchioloalveolar component, whereas the
\end{abstract}

Correspondence to: Professor Gabriella Fontanini, Section of Pathological Anatomy, Department of Surgery, University of Pisa, Via Roma 57, 56126 Pisa, Italy

E-mail: g.fontanini@do.med.unipi.it

Key words: EGFR mutations, laser capture microdissection, lung adenocarcinoma third mutated adenocarcinoma had a papillary subtype. Although we detected EGFR mutations only in 3 out of 62 adenocarcinomas and EGFR mutations were present in every subtype of each mutated adenocarcinoma, our research might represent a basis for further studies in characterizing molecular profiles of different component subtypes of lung adenocarcinoma.

\section{Introduction}

Adenocarcinoma of the lung is the most common histotype of non-small cell lung cancer in the USA and in Western countries (1). Lung adenocarcinoma is a heterogeneous entity because of its histologic features, molecular profiles and clinical behavior. Many histologic subtypes of lung adenocarcinoma have been described, and only recently has their importance in prognosis and response to therapy emerged.

To date, laser capture microdissection has been used mainly as a powerful tool to selectively isolate cancer cells from the surrounding normal tissue in order to avoid crosscontamination and masking of cancer-specific features by normal material. Such an application of laser microdissection technique has been reported in many research studies, including several investigating epidermal growth factor receptor $(E G F R)$ gene mutations in non-small cell lung cancer $(2,3)$, pancreatic adenocarcinoma $(4,5)$ and colon cancer $(6,7)$. However, besides being useful for separating cancer cells from the surrounding normal tissue, laser microdissection may greatly aid in isolating cancer cells belonging to distinct subtypes of lung adenocarcinomas in order to describe the molecular features specific to each. Since most adenocarcinomas are, in fact, 'mixed subtype', i.e. composed of more than one histologic subtype, isolation of DNA, RNA or protein from the whole tumor and not from microdissected subtypes might introduce bias when profiling cancers.

Characterization of EGFR mutations in distinct histologic subtypes of lung adenocarcinoma is becoming an important issue. Recently, mutations in the EGFR gene have been described in lung adenocarcinomas and have been associated with response to $E G F R$ tyrosine kinase inhibitors (TKI), erlotinib and gefitinib (8-10). EGFR mutations occur in 
approximately $30 \%$ of adenocarcinomas (11). Expression of the two most common EGFR mutations, exon 19 deletions and exon 21 L858R substitution, which lead to lung adenocarcinomas in mouse model systems $(12,13)$, is associated with a high response rate to erlotinib, a long progressive-free survival and a trend toward improved overall survival in humans (14). However, active research is underway to determine whether longer survival is due to a favorable prognosis of tumors harboring $E G F R$ mutations, treatment with erlotinib/gefitinib, or both. Associations between histology and EGFR mutations are still a matter of debate. In early studies of EGFR TKI, a higher response rate was observed in tumors classified as bronchioloalveolar carcinomas (BAC) or in patients with adenocarcinomas with bronchioloalveolar subtype or with clinical features associated with BAC, such as the absence of or more modest cigarette smoking $(8,15-17)$. Since then, investigators began to readdress the importance of pathologic subtypes, particularly bronchioloalveolar. However, recently, Motoi et al revealed that EGFR mutations seem to be strongly correlated with a papillary subtype and not with a bronchioloalveolar subtype (18).

In light of these findings, the goal of our study was to contribute to the characterization of distinct histologic subtypes of lung adenocarcinoma. We isolated, using laser capture microdissection, all subtypes composing 62 lung adenocarcinomas and subsequently analyzed $E G F R$ mutations in exons 18-21.

\section{Materials and methods}

Patients, tissues and histopathologic analyses. A retrospective analysis of 208 patients, who consecutively underwent surgical resection for lung tumors at the Department of Cardio-Thoracic Surgery and who were diagnosed with nonsmall cell lung cancer at the Section of Anatomic Pathology of the University of Pisa from January 1995 to December 1996, was conducted. Tumors, originally classified as squamous cell carcinomas, adenocarcinomas, bronchioloalveolar carcinomas and anaplastic large-cell carcinomas, were reclassified according to the 2004 World Health Organization (WHO) pathologic criteria (19) by two pathologists (V.D. and G.F.), and 62 cases of lung adenocarcinoma were identified. Special attention was paid to determine the different histologic subtypes/patterns of lung adenocarcinoma (mucinous/non-mucinous/mixed bronchioloalveolar, papillary, acinar, solid, signet ring, mucinous and clear cell). For all 62 adenocarcinomas, both formalin-fixed paraffin-embedded (FFPE) and frozen tissues were available. At least three FFPE tissue blocks of each adenocarcinoma were retrieved from archives. The most representative tissue block, for which all of the characteristic patterns of that specific neoplasm were present, was selected for each case. Frozen tissues from both the tumor and collateral normal lung were obtained immediately after surgery, before fixing tissues in $10 \%$ buffered formalin. Fresh tissues were isolated by pathologists, placed in sterile cryo-vials, snap-frozen in liquid nitrogen and stored at $-80^{\circ} \mathrm{C}$.

Clinical and pathologic features, including patient gender, age, smoking history, tumor histologic subtype, grade and stage, local recurrences, distant metastasis and overall survival were established. Fifty-eight patients were males and 4 females, with a median age of 67 years (range, 48-74 years). Follow-up was updated in June 2007 and ranged from 1 to 150 months (average follow-up, 38 months).

Laser capture microdissection and DNA extraction. Laser capture microdissection of each histologic subtype present in each case of lung adenocarcinoma was performed by a pathologist (V.D.) using the laser-assisted SL-Microcut MMI SmartCut [Molecular Machines \& Industries AG (MMI), Glattbrugg, Switzerland, distributed by Nikon, Florence, Italy]. The MMI SmartCut consists of an inverted Nikon TE 200 microscope (Nikon Biomedical Instruments, NY, USA), equipped with a high precision $x y$-stage, a solid state laser, a high resolution CCD camera, a computer and the specific software. The solid state laser has a wavelength of $355 \mathrm{~nm}$ and guarantees precise cutting without damaging the tissue. Sections $(5-\mu \mathrm{m})$ from each FFPE block were cut and placed on SL Microcut transfer film (Nikon). Sections were deparaffinized with xylene, rinsed in ethanol (100-70\%) and stained with hematoxylin and eosin. After staining, the slides were placed under a laminar hood for $5 \mathrm{~min}$ to dry. Immediately after drying, the LCM technique was performed. Care was taken in microdissecting cells from each single histologic subtype present. For each pattern, $\sim 10^{3}$ cells were microdissected. The microdissected samples were cleanly removed using the MMI Isolation Cap, which rests on the membrane and has no direct contact with the specimen. Images before cutting, after cutting and after capture (Fig. 1) were taken and stored. Isolation of genomic DNA from lasermicrodissected FFPE tissues of each histologic subtype composing each of the 62 lung adenocarcinomas was performed using the QIAamp DNA Micro Kit (Qiagen GmbH, Hilden, Germany). Tissues were lysed overnight in a humidified chamber at $56^{\circ} \mathrm{C}$ in $30 \mu 1$ of ATL buffer and $10 \mu 1$ of proteinase $\mathrm{K}$; after the overnight lysis, $10 \mu \mathrm{l}$ of ATL buffer was added. Carrier tRNA was added to improve DNA recovery. DNA was eluted in $30 \mu 1$ of distilled water twice, each time in a distinct microcentrifuge tube, and immediately processed for polymerase chain reaction amplification.

To confirm the results obtained from the FFPE specimens, isolation of genomic DNA from both normal and tumor snap-frozen tissues from 23 lung adenocarcinomas (3 adenocarcinomas harboring EGFR mutations in their patterns and 20 adenocarcinomas randomly selected out of the wild-type cases) was performed using the Nucleospin ${ }^{\circledR}$ Kit (MachereyNagel GmbH \& Co. KG, Düren, Germany) according to the manufacturer's instructions. For each case, $25 \mathrm{mg}$ of tissue was obtained by cutting frozen specimens using a cryostat (Micron, Walldone, Germany) pre-cooling at $-30^{\circ} \mathrm{C}$, and transferring them using sterile needles $(0.33$ x $12 \mathrm{~mm}, 29 \mathrm{G} \mathrm{x}$ $1 / 2$ inch) into microcentrifuge tubes containing $180 \mu \mathrm{l}$ of lysis buffer. DNA was eluted in $100 \mu 1$ of distilled water. The first and the last section cut from each specimen were stained with hematoxylin and eosin to confirm tissue histology.

Molecular analyses. Detection of EGFR mutations in exons 18-21 was performed using polymerase chain reaction singlestrand conformation polymorphism (PCR-SSCP) and direct DNA sequencing. 

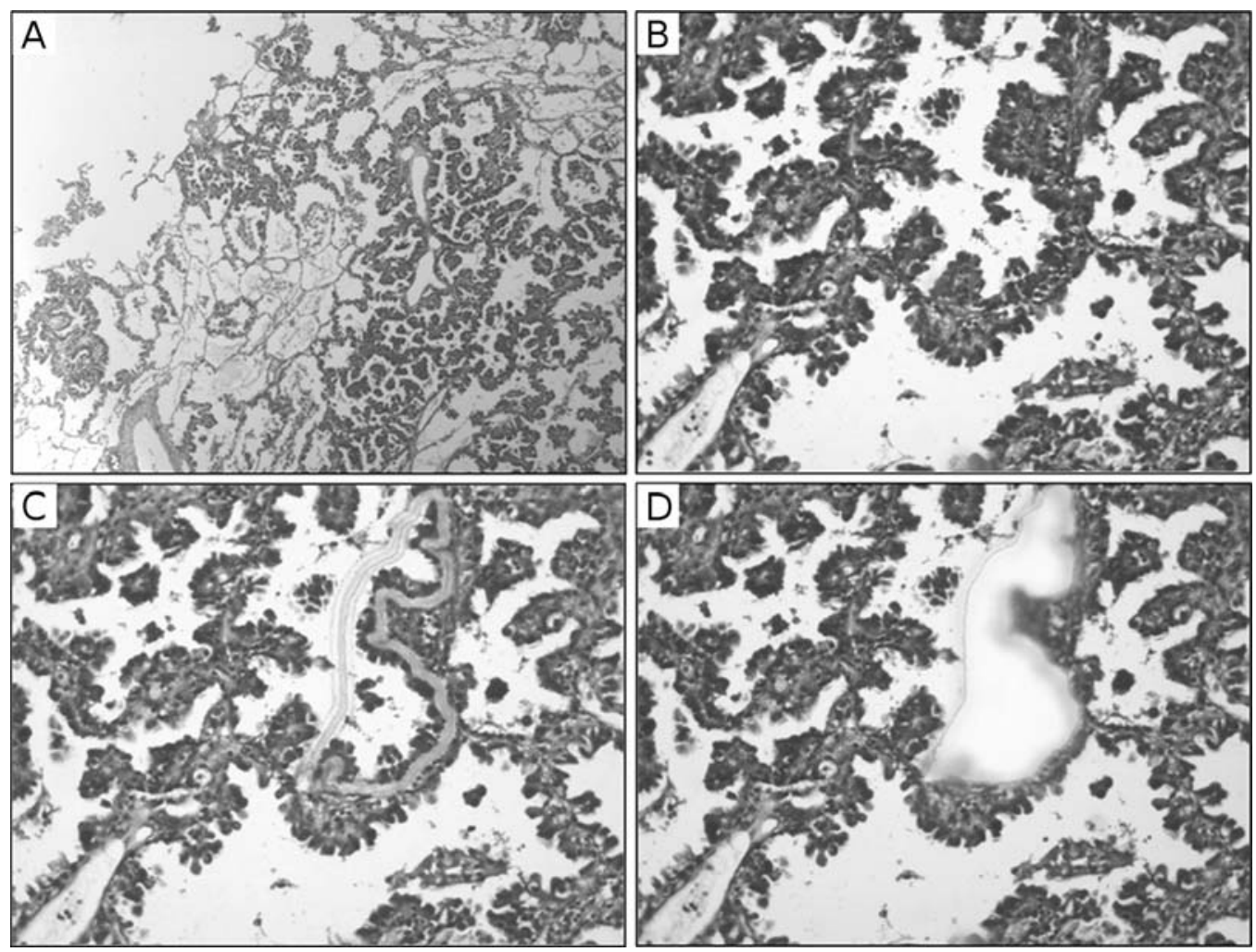

Figure 1. Laser capture microdissection of single patterns of lung adenocarcinoma. An adenocarcinoma of the lung with non-mucinous bronchioloalveolar pattern before laser microdissection (A, magnification $\mathrm{x} 4$; B, magnification $\mathrm{x} 20$ ), after laser cutting (C, magnification $\mathrm{x} 20$ ) and after tissue capture (D, magnification $\mathrm{x} 20$ ).

PCR-SSCP screening of EGFR mutations in all microdissected histologic patterns of lung adenocarcinoma was performed by amplifying exon 18 to 21 as follows. DNA was used as a template in a $50-\mu 1$ PCR mixture containing $10 \mathrm{mM}$ Tris- $\mathrm{HCl}, 50 \mathrm{mM} \mathrm{KCl}, 1.5 \mathrm{mM} \mathrm{MgCl}_{2}(\mathrm{pH} 8.3), 0.2 \mathrm{mM}$ dNTPs, 8 pmol of amplimers and $1.25 \mathrm{U}$ of AmpliTaq Gold DNA Polymerase (Applied Biosystems, Foster City, CA). The following PCR primers for EGFR exons 18-21 were used: for exon 18, 5'(Fw)-CTCTGTGTTCTTGTCCCCCC-3' and 5'(Rev)-GCCTGTGCCAGGGACCTTAC-3' (amplicon size, $166 \mathrm{bp})$; for exon 19, 5'(Fw)-GCACCATCTCACAA TTGCCAGTTA-3' and 5'(Rev)-GAGGTTCAGAGCCAT GGACCC-3' (amplicon size, $194 \mathrm{bp}$ ); for exon 20, 5'(Fw)CACACTGACGTGCCTCTCCCTCCC-3' and 5'(Rev)-CTC CCCTCCCGTATCTCCCTTCC-3' (amplicon size, 247 bp); for exon 21, 5'(Fw)-CCATGATGATCTGTCCCTCACA-3' and 5'(Rev)-AGGAAAATGCTGGCTGACCTAAAG-3' (amplicon size, 235 bp). A mock control in which no DNA was added was processed in parallel with each sample. Cycling conditions were as follows: initial denaturation $\left(94^{\circ} \mathrm{C}, 5 \mathrm{~min}\right)$, then 35 cycles of denaturation $\left(94^{\circ} \mathrm{C}\right.$ for $\left.40 \mathrm{sec}\right)$, annealing $\left(55^{\circ} \mathrm{C}\right.$ for $\left.40 \mathrm{sec}\right)$ and synthesis $\left(72^{\circ} \mathrm{C}\right.$ for $\left.40 \mathrm{sec}\right)$ followed by a final extension at $72^{\circ} \mathrm{C}$ for $5 \mathrm{~min}$. All PCR products were visualized by electrophoresis on a $1.5 \%$ agarose gel stained with ethidium bromide and purified using a PCR purification kit (Qiagen $\mathrm{GmbH}$ ). Purified products were then diluted 1:1 with denaturing solution ( $1 \%$ xylene cyanol, $1 \%$ bromophenol blue, $0.1 \mathrm{mM}$ EDTA and $99 \%$ formamide), denatured at $70^{\circ} \mathrm{C}$ for $5 \mathrm{~min}$ and immediately placed on ice to prevent annealing of the single-strand products. SSCP was carried out on a GenePhor Electrophoresis Unit using GeneGel Excel 12.5/24 (12.5\% T, 2\% C) (GE Healthcare Biosciences AB, Uppsala, Sweden), according to the manufacturer's instructions. Electrophoresis $(600 \mathrm{~V}, 25 \mathrm{~mA}, 15 \mathrm{~W})$ was performed at $16^{\circ} \mathrm{C}$ for $100 \mathrm{~min}$. Gels were stained with DNA Silver Staining Kit (GE Healthcare Biosciences AB), according to the manufacturer's instructions. Altered migrations patterns in two independent PCR-SSCP runs were indicative of DNA mutations. All purified PCR products were then sequenced by an ABI PRISM 3130 Genetic Analyzer (Applied Biosystems) according to the manufacturer's instructions. DNA sequences were compared to the wild-type sequences of EGFR exons 18-21 using the Basic Alignment Search Tool (BLAST) software available at the National Center for Biotechnology Information.

\section{Results}

Reclassification as lung adenocarcinomas according to 2004 WHO pathologic criteria. After a revision and reclassification of a two-year series of 208 consecutively resected non-small cell lung cancers according to the $2004 \mathrm{WHO}$ pathologic criteria, 62 adenocarcinomas of the lung were identified.

Only 37 out of the 62 cases $(59.7 \%)$ had been originally classified as 'adenocarcinomas'. In 14 cases (22.6\%) the original diagnosis was 'bronchioloalveolar carcinoma', whereas 6 cases $(9.7 \%)$ were originally classified as 'poorly differentiated squamous cell carcinoma' and 5 cases $(8.0 \%)$ as 'anaplastic large-cell carcinoma'. All 'bronchioloalveolar 


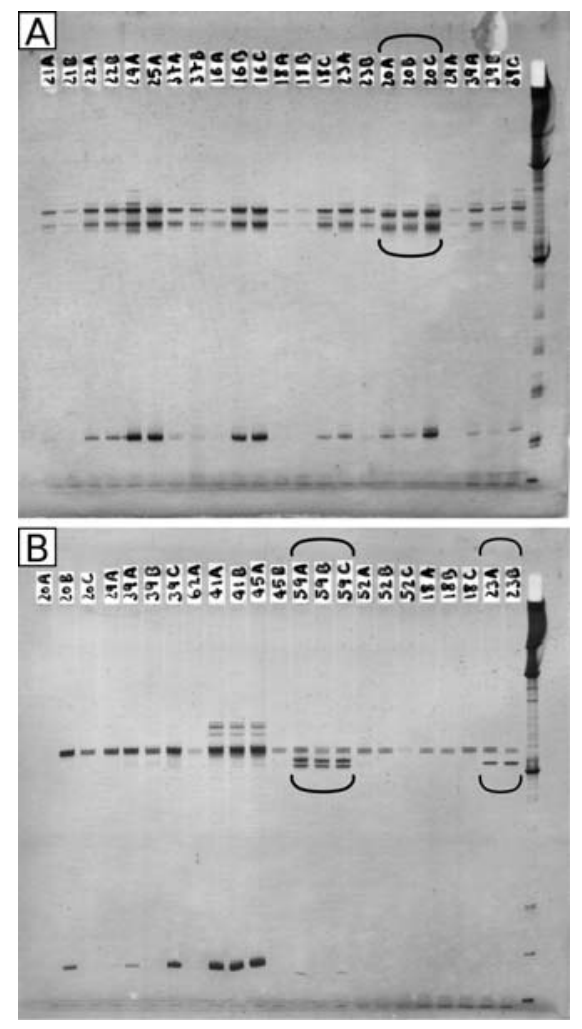

Figure 2. Detection of EGFR mutations in exons 19 and 21 using PCRSSCP. EGFR mutations in exon 19 and 21 in 3 lung adenocarcinomas and all their component histologic subtypes; altered migration patterns in PCRSSCP (DNAs isolated from FFPE laser-microdissected tissue). (A) Altered migration patterns of EGFR exon 21 L858R missense point mutation in patient ID\# 20; (B) altered migration patterns of EGFR exon 19 DEL E746T751 Ins VA (TTGCT) in patient ID\# 23 and altered migration patterns of EGFR exon 19 DEL LREAT (L747-T751) in patient ID\# 59.

carcinoma' were reclassified as 'adenocarcinomas with bronchioloalveolar features' due to the detection of several areas of invasion. Reclassification of 'poorly differentiated squamous cell carcinomas' and 'anaplastic large-cell carcinomas' as 'adenocarcinomas' was supported by microscopic diagnostic criteria and tumor immunophenotyping as reported in the 2004 WHO lung tumor classification (19). The differential diagnosis between 'poorly differentiated squamous cell carcinomas' and 'solid type adenocarcinomas' was based on the fact that the majority of squamous cell carcinoma express predominantly highmolecular-weight keratin (CK34ßE12) and very few express thyroid transcription factor-1 (TTF-1), which is a transcription factor that plays an important role in the lungspecific expression of surfactant proteins and is expressed in up to $75 \%$ of pulmonary adenocarcinomas. In our series, 6 tumors originally classified as "poorly differentiated squamous cell carcinomas' were immunostained for TTF-1 and CK34ßE12. Since they showed immunoreactivity for TTF-1 and were CK34ßE12-negative, they were reclassified as 'solid type adenocarcinomas'. The diagnosis of 'large-cell carcinomas' is a diagnosis of exclusion made after ruling out the presence of a component of adenocarcinoma or squamous cell carcinoma. Therefore, a tumor must be classified as 'solid type adenocarcinoma', when a minimum of 5 mucinous droplets are present in at least 2 high power fields or as

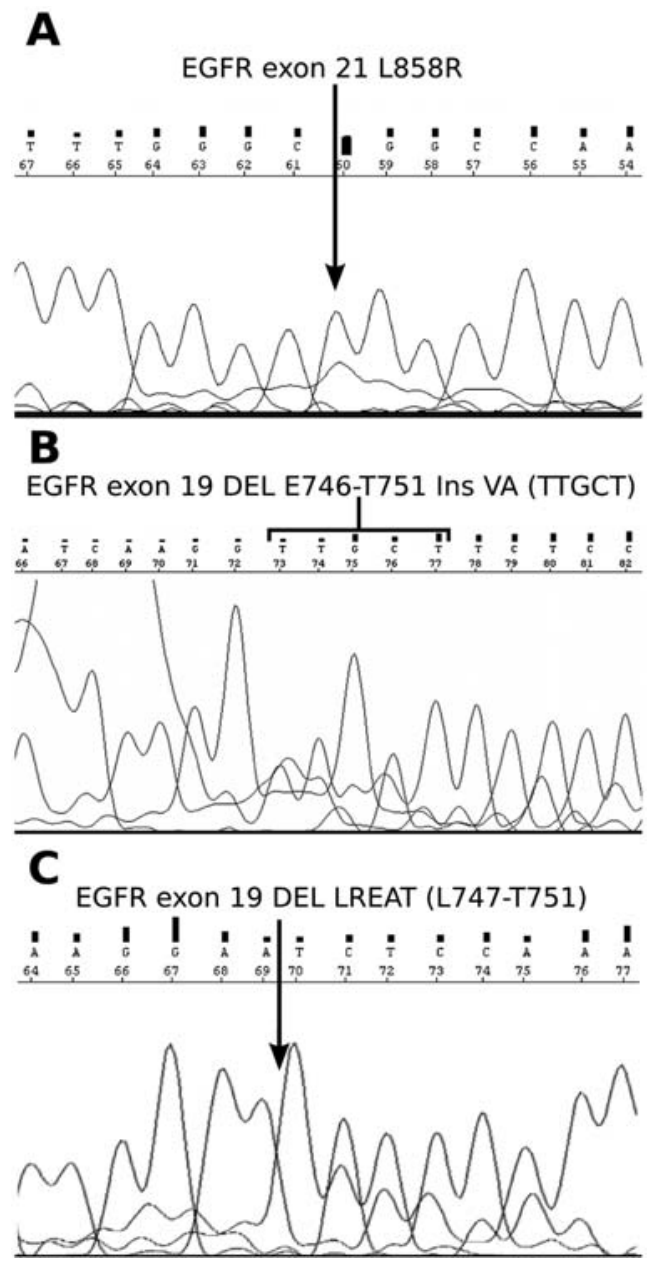

Figure 3. Electropherograms showing EGFR mutations in exons 19 and 21. Electropherograms showing EGFR exon 21 L858R missense point mutation in patient ID\# 20 (A), EGFR exon 19 DEL E746-T751 Ins VA (TTGCT) in patient ID\# 23 (B) and EGFR exon 19 DEL LREAT (L747-T751) in patient ID\# 59 (C).

'poorly differentiated squamous cell carcinoma' when foci of keratinization and/or intercellular bridges are present. The 5 cases originally classified as 'anaplastic large cell carcinomas' showed $>5$ mucinous droplets in at least 2 high power fields and were TTF-1-positive, so they were reclassified as 'solid type adenocarcinomas'.

Particular care was taken in identifying distinct histologic subtypes present in each adenocarcinoma. Twenty adenocarcinomas showed a single histologic pattern, which was 'non-mucinous bronchioloalveolar' in 8 cases, 'solid' in 6 cases, 'acinar' in 2 cases, 'mucinous' in 2 cases, 'mucinous bronchioloalveolar' in 1 case and 'clear cell' in 1 case. The remaining 42 adenocarcinomas with more then one component subtype ('mixed subtype') were composed of three patterns in 15 cases, two patterns in 25 cases and a mixture of nonmucinous bronchioloalveolar and mucinous bronchioloalveolar in 2 cases. In total, 119 component subtypes were identified: 38 'solid' patterns, 34 'acinar' patterns, 21 'bronchioloalveolar' patterns (17 'non-mucinous bronchioloalveolar' and 4 'mucinous bronchioloalveolar'), 9 'papillary' patterns, 9 'clear cell' patterns, 7 'mucinous' and 1 'signet ring'. 
Screening of EGFR mutations in exons 18-21 in histologic subtypes of lung adenocarcinoma. Detection of EGFR mutations in exons 18-21 was performed using PCR-SSCP and direct DNA sequencing in all 119 component subtypes of the 62 adenocarcinomas enrolled in the study. Laser capture microdissection of each specific pattern was performed by a pathologist (V.D.) and cross-contamination was avoided.

Only 3 adenocarcinomas (patient ID\# 20, 23 and 59) out of 62 harbored EGFR mutations: one (ID\# 20) in exon 21 and two in exon 19 (ID\# 23 and 59). Two out of 3 had a bronchioloalveolar component.

Patient ID\# 20 was a 62-year-old male, former smoker, originally diagnosed with BAC in two distinct lobes of the same lung (right superior lobectomy and right inferior atypical resection) and reclassified as 'adenocarcinoma with papillary, acinar and solid components'. All three component subtypes harbored EGFR exon 21 L858R $(2573 \mathrm{~T} \rightarrow \mathrm{G}$, $\mathrm{C} \underline{\mathrm{TG}} \rightarrow \mathrm{CGG}$ ) (Figs. 2A and 3A).

Patient ID\# 23 was a 75-year-old female, never smoker, originally diagnosed with a pT2N2 BAC (left pneumonectomy) and reclassified as 'adenocarcinoma with non-mucinous bronchioloalveolar and mucinous bronchioloalveolar components'. Both bronchioloalveolar component subtypes harbored an EGFR exon 19 E746-T751 deletion (2237-53) VA (TTGCT) insertion (Figs. 2B and 3B).

Patient ID\# 59 was a 68-year-old male, former smoker, originally diagnosed with a pT2N0 adenocarcinoma (right superior lobectomy) and reclassified as 'adenocarcinoma with non-mucinous bronchioloalveolar, acinar and solid components'. Even in this case all the component subtypes harbored the same EGFR mutation, which was the exon 19 LREAT deletion (15 bp, 2239-53, L747-T751) (Figs. 2B and 3C).

The above results were confirmed in DNAs isolated from frozen tumor tissues of the 3 adenocarcinomas harboring $E G F R$ mutations and from 20 randomly selected adenocarcinomas with wild-type EGFR exons 18-21 using PCRSSCP and direct sequencing. Normal lung tissue adjacent to adeno-carcinoma was available for 2 out of the 3 patients with EGFR mutations (ID\# 23 and 59) and for all the remaining 20 patients with wild-type $E G F R$ exons $18-21$. No $E G F R$ mutation was described in normal lung tissue from the 22 cases examined. Normal lung tissue was not available for patient ID\# 20 since the specimen which originally and macroscopically was thought to be normal was neoplastic in the first and last sections cut using the cryostat and stained with hematoxylin and eosin.

\section{Discussion}

Histologic heterogeneity of lung adenocarcinoma is well known and seems to be related to complex molecular profiles, different clinical behaviors and responsiveness or resistance to therapy. Many component subtypes have been described, and only recently has their importance in prognosis and response to therapy emerged. Thus, the goal of our study was to characterize EGFR mutations in distinct histologic subtypes of adenocarcinoma of the lung. To do so, we reviewed a complete two-year (1995-1996) series of 208 nonsmall cell lung cancers from our archives. Our accurate histologic revision according to the 2004 WHO histologic criteria led to the identification of 62 adenocarcinomas. Significantly, all the tumors (14 cases) originally diagnosed as 'BAC' were reclassified as adenocarcinomas with bronchioloalveolar component. Bronchioloalveolar carcinoma (BAC) is a subtype of adenocarcinoma characterized by lepidic growth of tumor cells along the alveoli without stromal, vascular, lymphatic or pleural invasion (19). Pure BAC is uncommon, accounting for $\sim 1-4 \%$ of NSCLC (20), whereas adenocarcinomas with a bronchioloalveolar component showing areas of invasion (referred to as 'adenocarcinomas with bronchioloalveolar features' or 'adenocarcinomas, mixed subtype with a bronchioloalveolar component') are very common and represent $\sim 50 \%$ of adenocarcinomas (19). In our series, 19 adenocarcinomas (30.6\%) had a bronchioloalveolar component. Another significant aspect of our review was the reclassification of 6 poorly differentiated squamous cell carcinomas and 5 anaplastic large-cell carcinomas as adenocarcinomas. As previously stated, immunoprofiling of these tumors using immunostaining for TTF-1 and CK34ßE12 was of great importance and helped in the differential diagnosis when we were not able to determine a histotype based only on morphological features.

Since the goal of our study was to characterize EGFR mutations in exons 18-21 in distinct subtypes of lung adenocarcinomas, particular care was taken in identifying every histologic subtype present in each adenocarcinoma. According to the literature, most of the adenocarcinomas analyzed were 'mixed subtype' (42 out of 62,67.7\%), mainly characterized by two subtypes. Among the 20 adenocarcinomas with a single histologic pattern, $250 \%$ was represented by tumors with a bronchioloalveolar component (9 cases). This evidence is important considering the fact that in many studies lung adenocarcinomas with bronchioloalveolar features are reported to be those affected by the highest number of EGFR mutations and the most responsive to TKI. Regarding the total number of subtypes, we identified 119 component subtypes in 62 adenocarcinomas with a prevalence of 'solid', 'acinar' and 'bronchioloalveolar' subtypes.

Mutations in EGFR exons 18-21, coding for the tyrosine kinase domain, have been extensively studied in lung adenocarcinomas since $2004(8,9)$. Mutations associated with responsiveness or resistance to therapy with TKI have been identified, and only recently have investigators begun to relate them to histologic subtypes. In our study, in order to accurately analyze EGFR exons 18-21 profile of each subtype and avoid cross-contamination, laser capture microdissection technique was used. In research, laser microdissection has been extensively used to selectively isolate malignant from normal cells, even in studies involving the molecular characterization of EGFR mutations in non-small cell lung cancer $(2,3)$. However, as far as we know, this is the first application of laser capture microdissection technique to isolate malignant cells from different histologic subtypes of lung adenocarcinoma in the same lung adenocarcinoma.

Only 3 out of the 62 adenocarcinomas analyzed harbored EGFR mutations. This low percentage (4.8\%) of cases with EGFR mutations compared to that reported in the literature (30\%) (11) might be explained by the unbalanced male to female ratio in our unselected series of patients (58 males 
and 4 females). In fact, as known, EGFR mutations are more frequently observed in women and non-smokers. Notably, 1 of the 3 patients harboring EGFR mutation was a neversmoker woman.

The three mutations we observed were either in exon 19 [E746-T751 deletion (2237-53) VA (TTGCT) insertion in ID\# 23 and LREAT deletion in ID\# 59] or in exon 21 [L858R (2573 $\mathrm{T} \rightarrow \mathrm{G}, \mathrm{C} \underline{\mathrm{T}} \rightarrow \mathrm{C} \underline{\mathrm{GG}})$ ]. The exon $19 \mathrm{LREAT}$ deletion and exon $21 \mathrm{~L} 858 \mathrm{R}$ point mutation are the most common EGFR mutations and are associated with responsiveness to TKI. L858R (substitution mutations changing leucine 858 to arginine) lays in the activation and glycine-rich $\mathrm{P}$ loops, which are important for autoregulation, and multiple deletion mutations clustering in the region spanning codons 746-750 (ELREA) are around the active site of the kinase $(8,9)$. Somatic mutations around the EGFR tyrosine kinase domain enhance ligand-inducing EGFR autophosphorylation and confer increased sensitivity to gefitinib.

In our study, EGFR mutations were observed in all subtypes composing the same neoplasm. This suggests that, in patients with lung adenocarcinoma, EGFR mutations are not associated with particular histologic subtypes and probably occur at an early stage of tumorigenesis. However, the proportion of mutated cases in our study was too low to confirm this. Notably, 2 out of the 3 mutated adenocarcinomas had a bronchioloalveolar component, partly confirming that the bronchioloalveolar pattern is frequently associated with EGFR mutations, whereas the third mutated adenocarcinoma had a papillary subtype. In a recent study, Motoi et al examined in detail different histologic subtypes in 100 lung adenocarcinomas and found that it is important to include the 'major histologic subtype' in the scheme of classification of 'mixed subtype adenocarcinomas'. In their comprehensive study, the papillary pattern, which was the most common major histologic subtype (37\%), strongly correlated with EGFR mutations and a gene profile cluster (cluster 1), was also associated with less smoking. In contrast, the solid pattern, which was the third most common major histologic subtype (25\%) after papillary and acinar (30\%), strongly correlated with a less favorable patient survival and a gene profile cluster strongly associated with heavier smoking, larger tumor size and higher grade (18).

In conclusion, although we detected EGFR mutations only in 3 out of the 62 adenocarcinomas, and EGFR mutations were present in every subtype of each mutated adenocarcinoma, our research represents a basis for further studies in characterizing molecular profiles of different subtypes composing single lung adenocarcinomas. We believe that the novel application of laser capture microdissection technique described in our study might be a powerful and helpful tool for molecular characterization of single histologic subtypes of lung adenocarcinoma. From molecular analyses of laser capture microdissected histologic subtypes of lung adenocarcinoma, we may better define their genetic profiles and recognize unforeseen histologic-genetic correlations useful to a comprehensive approach to histologic subclassification of lung adenocarcinoma and to design new tailored therapies. Thus, we are conducting further studies of EGFR mutations in distinct histologic subtypes laser capture-microdissected from a larger, pre-selected and prospectively acquired series of adenocarcinomas harboring $E G F R$ mutations in exon 18-21, for which data on response to therapy with TKI are available. Given the histologic heterogeneity of lung adenocarcinoma and since the major genetic pathways of $30-70 \%$ of lung adenocarcinomas need to be discovered, a comprehensive approach in histologic subtyping might provide an advantage in the recognition of correlations between histology and genetics and in the identification of prognostic and predictive markers in such complex tumors.

\section{Acknowledgements}

We thank AIRC (Associazione Italiana per la Ricerca sul Cancro) for supporting our research.

\section{References}

1. Travis WD, Travis LB and Devesa SS: Lung cancer. Cancer 75: 191-202, 1995.

2. Pugh TJ, Bebb G, Barclay L, et al: Correlations of EGFR mutations and increases in EGFR and HER2 copy number to gefitinib response in a retrospective analysis of lung cancer patients. BMC Cancer 7: 128, 2007.

3. Rosell R, Ichinose $\mathrm{Y}$, Taron M, et al: Mutations in the tyrosine kinase domain of the EGFR gene associated with gefitinib response in non-small-cell lung cancer. Lung Cancer 50: 25-33, 2005.

4. Immervoll H, Hoem D, Kugarajh K, et al: Molecular analysis of the EGFR-RAS-RAF pathway in pancreatic ductal adenocarcinomas: lack of mutations in the BRAF and EGFR genes. Virchows Arch 448: 788-796, 2006.

5. Tzeng CW, Frolov A, Frolova N, et al: Epidermal growth factor receptor (EGFR) is highly conserved in pancreatic cancer. Surgery 141: 464-469, 2007

6. Yang D, Schneider S, Azuma M, et al: Gene expression levels of epidermal growth factor receptor, survivin, and vascular endothelial growth factor as molecular markers of lymph node involvement in patients with locally advanced rectal cancer. Clin Colorectal Cancer 6: 305-311, 2006

7. Nagahara H, Mimori K, Ohta M, et al: Somatic mutations of epidermal growth factor receptor in colorectal carcinoma. Clin Cancer Res 11: 1368-1371, 2005.

8. Lynch TJ, Bell DW, Sordella R, et al: Activating mutations in the epidermal growth factor receptor underlying responsiveness of non-small-cell lung cancer to gefitinib. N Engl J Med 350: 2129-2139, 2004

9. Paez JG, Janne PA, Lee JC, et al: EGFR mutations in lung cancer: Correlation with clinical response to gefitinib therapy. Science 304: 1497-1500, 2004.

10. Pao W, Miller V, Zakowski M, et al: EGF receptor gene mutations are common in lung cancers from 'never smokers' and are associated with sensitivity of tumors to gefitinib and erlotinib. Proc Natl Acad Sci USA 101: 13306-13311, 2004.

11. Shigematsu H and Gazdar AF: Somatic mutations of epidermal growth factor receptor signaling pathway in lung cancers. Int $\mathrm{J}$ Cancer 118: 257-262, 2006.

12. Politi K, Zakowski MF, Fan PD, Schonfeld EA, Pao W and Varmus HE: Lung adenocarcinomas induced in mice by mutant EGF receptors found in human lung cancers respond to a tyrosine kinase inhibitor or to down-regulation of the receptors. Genes Dev 20: 1496-1510, 2006.

13. Ji H, Li D, Chen L, et al: The impact of human EGFR kinase domain mutations on lung tumorigenesis and in vivo sensitivity to EGFR-targeted therapies. Cancer Cell 9: 485-495, 2006.

14. Miller VA, Riely GJ, Zakowski MF, et al: Molecular characteristics of bronchioloalveolar carcinoma and adenocarcinoma, bronchioloalveolar carcinoma subtype, predict response to erlotinib. J Clin Oncol 26: 1472-1478, 2008.

15. Kris MG, Natale RB, Herbst RS, et al: Efficacy of gefitinib, an inhibitor of the epidermal growth factor receptor tyrosine kinase, in symptomatic patients with non-small cell lung cancer: A randomized trial. JAMA 290: 2149-2158, 2003.

16. Fukuoka M, Yano S, Giaccone G, et al: Multi-institutional randomized phase II trial of gefitinib for previously treated patients with advanced non-small-cell lung cancer (The IDEAL 1 Trial) (corrected). J Clin Oncol 21: 2237-2246, 2003. 
17. Miller VA, Kris MG, Shah N, et al: Bronchioloalveolar pathologic subtype and smoking history predict sensitivity to gefitinib in advanced non-small-cell lung cancer. J Clin Oncol 22: 1103-1109, 2004

18. Motoi N, Szoke J, Riely GJ, et al: Lung adenocarcinoma: Modification of the 2004 WHO mixed subtype to include the major histologic subtype suggests correlations between papillary and micropapillary adenocarcinoma subtypes, EGFR mutations and gene expression analysis. Am J Surg Pathol 32: $810-827,2008$
19. Travis WD, Brambilla E, Muller-Hermelink HK and Harris CC: World Health Organization Classification of Tumours Pathology and Genetics of Tumours of the Lung, Pleura, Thymus and Heart. IARC Press, Lyon, 2004

20. Read WL, Page NC, Tierney RM, Piccirillo JF and Govindan R: The epidemiology of bronchioloalveolar carcinoma over the past two decades. Analysis of the SEER database. Lung Cancer 45: $137-142,2004$ 